This item was submitted to Loughborough's Research Repository by the author.

Items in Figshare are protected by copyright, with all rights reserved, unless otherwise indicated.

\title{
Quasiparticle lifetimes in the charged Bose gas and the cuprates
}

PLEASE CITE THE PUBLISHED VERSION

\section{LICENCE}

CC BY-NC-ND 4.0

\section{REPOSITORY RECORD}

Alexandrov, A.S., and C.J. Dent. 2019. "Quasiparticle Lifetimes in the Charged Bose Gas and the Cuprates". figshare. https://hdl.handle.net/2134/1156. 
This item was submitted to Loughborough's Institutional Repository (https://dspace.lboro.ac.uk/) by the author and is made available under the following Creative Commons Licence conditions.

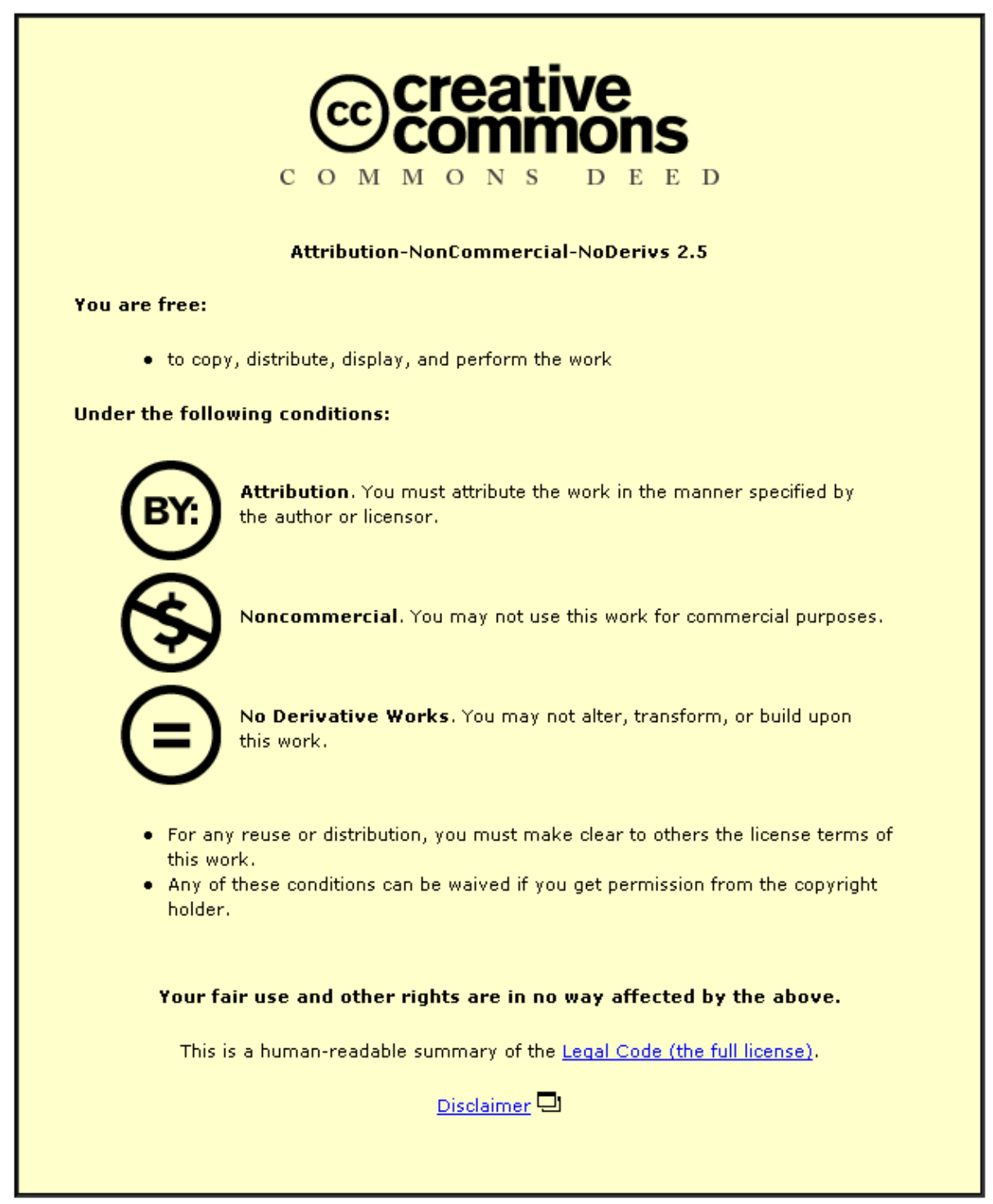

For the full text of this licence, please go to: http://creativecommons.org/licenses/by-nc-nd/2.5/ 


\title{
Quasiparticle lifetimes in the charged Bose gas and the cuprates
}

\author{
A.S. Alexandrov and C. J. Dent \\ Department of Physics, Loughborough University, Loughborough LE11 3TU, U.K.
}

The scattering cross section of a Coulomb potential screened by a charged Bose gas (CBG) is calculated both above and below the Bose-Einstein condensation temperature, using the variable phase method. In contrast with the BCS superconductor, the screened scattering potential and quasiparticle lifetime are found to be very different in the superconducting and normal states. We apply the result to explain the appearence of a sharp peak in the ARPES spectra in some cuprates below the superconducting transition.

There is a growing body of evidence that cuprate superconductivity is due to the condensation of bipolarons, local bosonic pairs of carriers bound by the strong electron-phonon interaction [1]. The theory has been applied to explain the upper critical field [2], magnetic susceptibility [3], anisotropy [4], isotope effect on the supercarrier mass [5] and the pseudogap [1,6,6]. It provides a parameter-free formula for the superconducting $T_{c}$ and a parameter-free fit to the electronic specific heat near the transition [9]. The d-wave order parameter and the single particle tunneling density of states can be understood in the framework of Bose-Einstein condensation of inter-site bipolarons as well [10,11]. We have also explained various features of the data from angle-resolve photoemission spectroscopy (ARPES) 12. We assumed that a single photoexcited hole in the oxygen band is scattered by impurities, while the chemical potential is pinned inside the charge-transfer (optical) gap due to bipolaron formation. The normal state gap, the spectral shape and the polarisation dependence of the ARPES spectra were well described within this approach in a few cuprates. Recently it has been observed at certain points in the Brillouin zone that the ARPES peak in the bismuth cuprates is relatively sharp at low temperatures in the superconduncting state, but that it almost disappears into the background above the transition [17,18].

In this letter, we first calculate the scattering cross section of single-particle excitations off a Coulomb scattering centre in the charged Bose gas, both above and below the Bose-Einstein condensation temperature. We then propose that the appearence of a sharp ARPES peak below the transition in the cuprates is caused by a large increase in the quasiparticle lifetime due to condensate screening of scatterers.

First we calculate the scattering cross-section of a charged particle (mass $m$, charge $e$ ) scattered by a static Coulomb potential $V(r)$ screened by the CBG. The gen- eral theory of potential scattering in terms of phase shifts was developed in the earliest days of quantum mechanics (see for instance 13.). While in principle this allows scattering cross sections to be calculated for an arbitary potential, in practice the equations for the radial part of the wavefunction may only be solved analytically for a few potentials, and in the standard formulation are not in a suitable form for numerical computation. The 'variable phase' approach [14] solves this problem by making the phase shifts functions of the radial coordinate, and then the Schrodinger equation for each radial component of the wavefunction reduces to a first order differential equation for the corresponding phase shift.

In dimensionless units $(\hbar=2 m=1)$, the Schrodinger equation for the radial part of the angular momentum $l$ component of the wavefunction of a particle with wavevector $k$ undergoing potential scattering is

$$
u_{l}^{\prime \prime}(r)+\left[k^{2}-l(l+1) / r^{2}-V(r)\right] u_{l}(r)=0 .
$$

The scattering phase shift $\delta_{l}$ is obtained by comparison with the asymptotic relation

$$
u_{l}(r) \stackrel{r \rightarrow \infty}{\longrightarrow} \sin \left(k r-l \pi / 2+\delta_{l}\right),
$$

and the scattering cross section is then

$$
\sigma=\frac{4 \pi}{k^{2}} \sum_{l=0}^{\infty} \sin ^{2} \delta_{l}
$$

In the variable phase method [14, we must satisfy the condition that

$$
V(r) \stackrel{r \rightarrow 0}{\longrightarrow} V_{0} r^{-n}
$$

with $n<2$. The angular momentum $l$ phase shift is then

$$
\delta_{l}=\lim _{r \rightarrow \infty} \delta_{l}(r)
$$

where the phase function $\delta_{l}(r)$ satisfies the phase equation

$$
\delta_{l}^{\prime}(r)=-k^{-1} V(r)\left[\cos \delta_{l}(r) \hat{j}_{l}(k r)-\sin \delta_{l}(r) \hat{n}_{l}(k r)\right]^{2},
$$

with

$$
\delta_{l}(r) \stackrel{r \rightarrow 0}{\longrightarrow}-\frac{V_{0} r^{-n}}{k^{2}} \frac{(k r)^{2 l+3}}{(2 l+3-n)[(2 l+1) ! !]^{2}},
$$

and $j_{l}(x)$ and $n_{l}(x)$ are the Riccati-Bessel functions [14]. In the $l=0$ case, the phase equation reduces to 


$$
\delta_{0}^{\prime}(r)=-k^{-1} V(r) \sin ^{2}\left[k r+\delta_{0}(r)\right] .
$$

In the slow particle limit, we may also neglect higher order contributions to the scattering cross section, so that

$$
\sigma=\frac{4 \pi}{k^{2}} \sin ^{2} \delta_{0}
$$

The effective potential about a point charge in the CBG was calculated by Hore and Frankel [15]. The static dielectric function of the CBG is:

$$
\epsilon(\vec{q}, 0)=1+\sum_{\vec{p}} \frac{4 \pi\left(e^{*}\right)^{2}}{q^{2} \Omega}\left(\frac{F_{0}(\vec{p})-F_{0}(\vec{p}-\vec{q})}{-\left(1 / m_{b}\right) \vec{p} \cdot \vec{q}+q^{2} / 2 m_{b}}\right),
$$

in which $e^{*}=2 e$ the boson charge, and $F_{0}(\vec{p})=$ $\left(e^{\left(p^{2} / 2 m_{b}-\mu\right) / k_{B} T}-1\right)^{-1}$, the Bose distribution function. It has been shown [16] that Eq. 10 is valid even beyond the simplest random phase appeoximation assumed in Ref. 15]. Eliminating the chemical potential, for small $q$ the dielectric function for $T<T_{c}$ is

$$
\epsilon(\vec{q}, 0)=1+\frac{4 m_{b}^{2} \omega_{p}^{2}}{q^{4}}\left[1-\left(\frac{T}{T_{c}}\right)^{3 / 2}\right]+O\left(\frac{1}{q^{3}}\right),
$$

and for $T \rightarrow \infty$ is

$\epsilon(\vec{q}, 0)=1+\frac{1}{q^{2}} \frac{m_{b} \omega_{p}^{2}}{k_{B} T}\left[1+\frac{\zeta\left(\frac{3}{2}\right)}{2^{3 / 2}}\left(\frac{T_{c}}{T}\right)^{3 / 2}+\ldots\right]+O\left(q^{0}\right)$,

with $\omega_{p}^{2}=4 \pi\left(e^{*}\right)^{2} \rho / m_{b}$, and $\rho$ the boson density. If the unscreened scattering potential is the Coulomb potential $V(r)=V_{0} / r$, then performing the inverse Fourier transforms, one finds that for $T<T_{c}$ [15]

$$
\lim _{r \rightarrow \infty} V(r)=\frac{V_{0}}{r} \exp \left[-K_{s} r\right] \cos \left[K_{s} r\right] \equiv V_{s}(r)
$$

with

$$
K_{s}=\left(m_{b}^{2} \omega_{p}^{2}\left[1-\left(\frac{T}{T_{c}}\right)^{3 / 2}\right]\right)^{1 / 4},
$$

and for $T \rightarrow \infty$,

$$
\lim _{r \rightarrow \infty} V(r)=\frac{V_{0}}{r} \exp \left[-K_{n} r\right] \equiv V_{n}(r)
$$

with

$$
K_{n}=\left(\frac{m_{b} \omega_{p}^{2}}{k_{B} T}\right)^{1 / 2}\left[1+\frac{\zeta\left(\frac{3}{2}\right)}{2^{3 / 2}}\left(\frac{T_{c}}{T}\right)^{3 / 2}+\ldots\right]^{1 / 2} .
$$

The $T<T_{c}$ result is exact for all $r$ at $T=0$.

There are two further important analytical results; the first (Levinson's Theorem [14]) states that for 'regular' potentials (which include all those which we shall be concerned with), the zero-energy phase shift is equal to $\pi$ multiplied by the number of bound states of the potential. The second is the well-known Wigner resonance scattering formula [13], states that for slow particle scattering of a particle with energy $E$ off a potential with a shallow bound state of binding energy $\epsilon \lesssim E$ the total scattering cross section is

$$
\sigma=\frac{2 \pi}{m} \frac{1}{E+|\epsilon|}
$$

We have used this to check that our calculation method works correctly by comparing our results with Eq. 17 for various potentials with shallow bound states (Fig. 1).

The zero-energy scattering cross-sections for the potentials $V_{n}(r)=-\left(V_{0} / r\right) e^{-K r}$ and $V_{s}(r)=$ $-\left(V_{0} / r\right) e^{-K r} \cos (K r)$ are shown in Fig. 2a. These graphs are plotted for $V_{0}=1$; in each case, the equivalent graph for arbitary $V_{0}$ may be found by rescaling $\sigma$ and $K$. According to the Wigner formula (Eq. 17), as $K$ is decreased, when a new bound state appears there should be a peak in the cross-section, as there will then be a minimum in the binding energy of the shallowest bound state. This is the origin of the peaks in Fig. 2a, which may be checked using Levinson's Theorem. It can also be seen that as $K$ is decreased, the first few bound states appear at higher $K$ in the ordinary Yukawa potential; this agrees with the intuitive conclusion that the bound states should in general be deeper in the non-oscillatory potential. Another intuitive expectation which is also bourne out is that for a given $V_{0}$ and $K$, the non-oscillatory potential should be the stronger scatterer; in Fig. 2b it may be seen that this is the case when $K$ is large enough for neither potential to have bound states (the difference in cross sections is then in fact about three orders of magnitude.)

Now we address the possible application of our results to the ARPES linewidth in the cuprates. According to [12], the ARPES peak is related to photoexctited holes with small group velocity near the top of the oxygen band. The quantities necessary in order to calculate the scattering cross section of impurities (the dopants) in the cuprates are the static dielectric constant, the effective mass of the bipolaronic carriers, the charge on the scattering centres and the bipolaron density. The situation is however complicated by the anisotropy of the effective mass tensor. The value of the effective mass of the bipolarons in the cuprates is readily found from the penetration depth [8]. In BSCCO the in-plane bipolaron mass $m_{b}$ is about $5-6 m_{e}$. The dopants in $\mathrm{Bi}_{2} \mathrm{Sr}_{2} \mathrm{CaCu}_{2} \mathrm{O}_{8}+\delta$ are $\mathrm{O}^{2-}$ ions, and thus the Coulomb potential between a scattering centre and a hole is $V(r)=-2 e^{2} /\left(\epsilon_{0} r\right)$. The issue of the dielectric constant is more contentious; measurements suggest that it may be as high as 1000 [19. The variable phase method has only been derived for the isotropic problem, so we cannot apply our theory to make 
a quantitative conclusion about the quasiparticle lifetime at different temperatures in the cuprates. However, we can provide an important general conclusion about the relative value of the cross-sections in the normal and superconducting states.

At zero temperature, the screening wavevector is $K_{0}=$ $\left(m_{b} \omega_{p}\right)^{1 / 2}$, and at a temperature $\alpha T_{c}$ well above the transition, it is $K_{\alpha T_{c}}=\left(m_{b} \omega_{p}^{2} / k_{B} \alpha T_{c}\right)^{1 / 2}$. Substituting $\omega_{p}$ and $k_{B} T_{c}=3.3 n^{2 / 3} / m_{b}$, we obtain:

$$
\frac{K_{\alpha T_{c}}}{K_{0}}=\left(\frac{2.1 e m_{b}^{1 / 2}}{\epsilon_{0}^{1 / 2} \rho^{1 / 6} \alpha}\right)^{1 / 2}
$$

From this, we see that the ratio is only marginally dependent on the boson density, so substituting for $\rho=$ $10^{21} \mathrm{~cm}^{-3}, e$, and $m_{e}$, we obtain

$$
\frac{K_{\alpha T_{c}}}{K_{0}}=3.0\left(\frac{\left(m_{b} / m_{e}\right)^{1 / 2}}{\epsilon_{0}^{1 / 2} \alpha}\right)^{1 / 2}
$$

With realistic boson masses and dielectic constants, $K_{\alpha T_{c}}$ and $K_{0}$, while different, are of the same order of magnitude. In the isotropic model, if the screening wavevectors are such that neither the normal state or condensate impurity potentials have bound states, with these parameters it would then follow that the quasiparticle lifetime is much greater in the superconducting state, Fig. 2b. We propose that this effect also occurs in the realistic non-isotropic model, and could then explain the appearence of a sharp ARPES peak in the superconducting state of BSCCO. With doping, the screening radius decreases both in the normal, Eq. 16, and superconducting states, Eq. 14. This explains another fascinating experimental observation, namely the strange doping dependence of the ARPES linewidth. Optimallly and overdoped cuprates, due to the higher carrier density, have shorter range scattering potentials with smaller crosssections compared with the underdoped cuprates.

In summary, we have calculated the scattering cross section of a Coulomb scattering centre in the charged Bose gas both above and below the condensation temperature. In contrast to the BCS superconductor, the scattering potential in the CBG is different in the normal and superconducting states. This is because the coherence length in the $\mathrm{CBG}$ is the same (at $T=0$ ) as the screening radius [20], while in the BCS superconductor it is a few orders of magnitude larger. We find that for the realistic parameters, the scattering cross section above $T_{c}$ in the bismuth cuprates might be around three orders of magnitude larger than at $T=0$. We propose that the appearence of a sharp peak in the ARPES spectra of BSCCO below the superconducting transition and its doping dependence is due to the condensate screening of the scattering potential. We acknowledge valuable discussions with M. Portnoi. CJD was supported financially in this work by the UK EPSRC.
[1] A.S. Alexandrov and N.F. Mott, Rep. Prog. Phys. 57 1197 (1994); 'High Temperature Superconductors and Other Superfluids' Taylor and Francis, London (1994); 'Polarons and Bipolarons', World Scientific, Singapore (1995).

[2] M.S. Osofsky et al, Phys. Rev. Lett. 71, 2315 (1993); ibid 72, 3292 (1994); D.D. Lawrie et al, J. Low. Temp. Phys., 107, 491 (1997); A.S. Alexandrov et al, Phys. Rev. Lett. 71, 983 (1996); V.F. Gantmacher et al, Zh. Eksp. Teor. Fiz. 115, 268 (1999) (JETP 88, 148 (1999); V.N. Zavaritsky et al, EuroPhys. Lett. 51, 334 (2000).

[3] K.A. Müller et al, J. Phys.: Condens. Matter 10, L291 (1998).

[4] J. Hofer et al, Physica C 297, 103 (1998).

[5] G. Zhao, M.B. Hunt, H. Keller and K.A. Müller, Nature 385, 236 (1997).

[6] D. Mihailovic, V.V. Kabanov, K. Zagar, J. Demsar, Phys. Rev. B 60, R6995 (1999).

[7] D. Rubio Temprano, J. Mesot, S. Janssen, K. Conder, A. Furrer, H. Mutka and K.A. Müller,, Phys. Rev. Lett. 84, 1990 (2000).

[8] A.S. Alexandrov, Phys. Rev. Lett. 82, 2620 (1999); A.S. Alexandrov and V.V. Kabanov, Phys. Rev. B 59, 13628 (1999).

[9] A.S. Alexandrov, W.H. Beere, V.V. Kabanov and W.Y. Liang, Phys. Rev. Lett., 79, 1551 (1997).

[10] A.S. Alexandrov, Physica C (Amsterdam) 305, 46 (1998).

[11] A.S. Alexandrov and A.F. Andreev, cond-mat/0010060.

[12] A.S. Alexandrov and C.J. Dent, Phys. Rev. B60, 15414 (1999).

[13] L.D. Landau and E.N. Lifshitz, 'Quantum Mechanics (Non-relativistic theory)', Third Edition, ButterworthHeinemann, Oxford (1977).

[14] F. Calogero, 'Variable Phase Approach to Potential Scattering', Academic Press, New York, 1967.

[15] S.R. Hore and N.E. Frankel, Phys. Rev. B12, 2619 (1975).

[16] A.S. Alexabdrov and W.H. Beere, Phys. Rev. B 51, 5887 (1995).

[17] A. Kaminski et al, Phys. Rev. Lett. 84, 1788 (2000).

[18] A.V. Fedorov et al, Phys. Rev. Lett. 82, 2179 (1999).

[19] Z.Zhai, P.V. Parmi, J.B. Sokoloff, S. Sridhar and A. Erb, cond-mat/0005535 and references therein; K.A. Müller, private communication.

[20] A.S. Alexandrov, Phys. Rev. B60, 14513 (1999).

\section{Figure Captures}

Fig. 1. Plot of scattering cross section $\sigma_{n}$ against scattered particle momentum $k$ for small $k$ (solid line) and fit using the Wigner formula (broken line) for potential $V_{n}(r)=(-1 / r) \exp (-0.55 r)$. There is good agreement between the numerical and Wigner results.

Fig. 2. (a) Plots of zero-energy scattering cross sections (i) $\sigma_{n}$ and (ii) $\sigma_{s}$ against screening wavevector 
$K$ for the potentials (i) $V_{n}(r)=-(1 / r) e^{-K_{n} r}$ and (ii) $V_{s}(r)=-(1 / r) e^{-K_{s} r} \cos \left(K_{s} r\right)$. (b) Plot of $\sigma_{n} / \sigma_{s}$ for a range of $K_{n}=K_{s}$ in which neither potential has any bound states. In each case the units are those used to derive the phase equation. 
arXiv:cond-mat/0012234 v1 13 Dec 2000

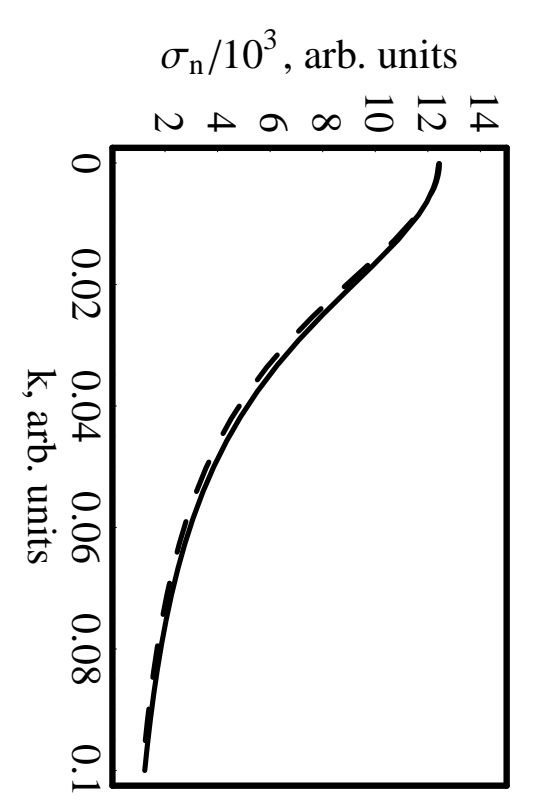




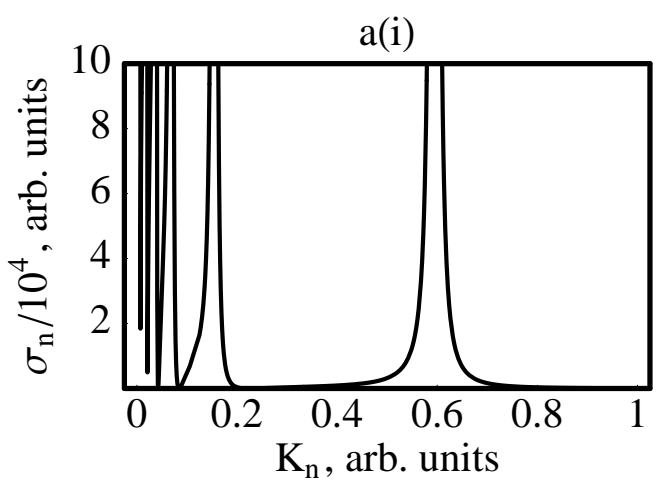

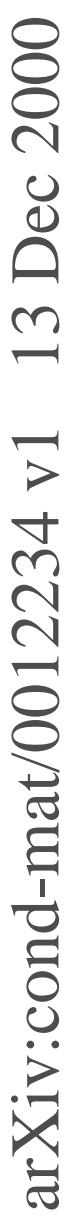
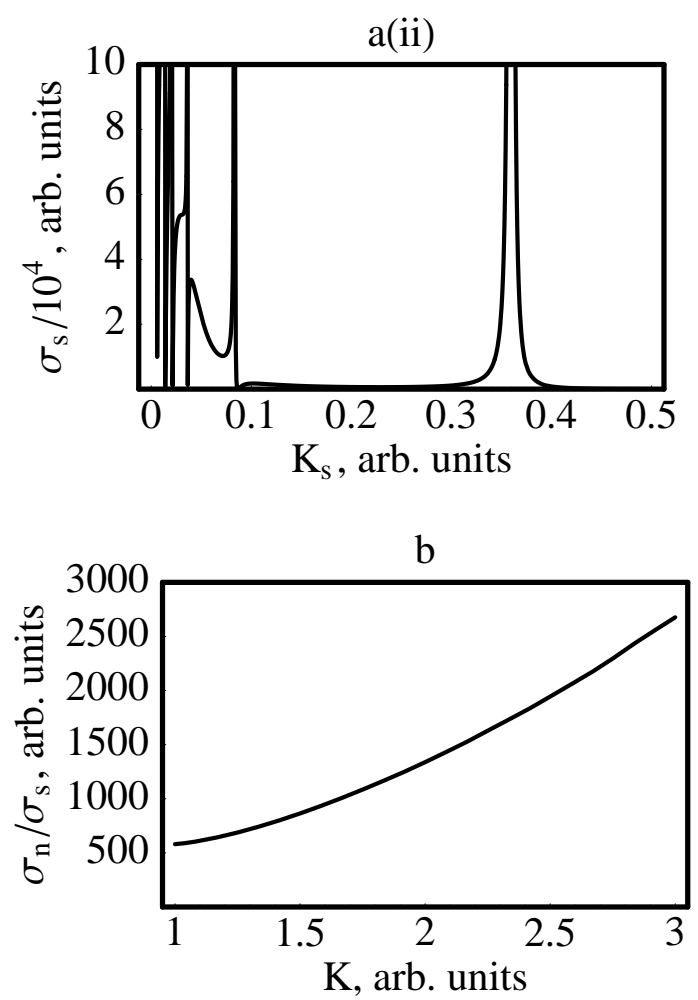